\title{
On the focusing conditions in time-reversed acoustics, seismic interferometry and Marchenko imaging
}

Kees Wapenaar*, Jan Thorbecke, Joost van der Neut, Delft University of Technology; Ivan Vasconcelos, Schlumberger Gould Research; Dirk-Jan van Manen, ETH Zürich; Matteo Ravasi, University of Edinburgh.

\section{SUMMARY}

Despite the close links between the fields of time-reversed acoustics, seismic interferometry and Marchenko imaging, a number of subtle differences exist. This paper reviews the various focusing conditions of these methods, the causality/acausality aspects of the corresponding focusing wavefields, and the requirements with respect to omnidirectional/singlesided acquisition.

\section{INTRODUCTION}

It has been noted by various authors that there exists a close link between time-reversed acoustics and seismic interferometry (Derode et al., 2003; Wapenaar et al., 2005; van Manen et al., 2005; Bakulin and Calvert, 2006). More recently, similar links have been discovered between seismic interferometry and autofocusing, also known as Marchenko imaging (Broggini and Snieder, 2012; Wapenaar et al. 2012). All these methods have in common that recorded wavefields are focused onto a point inside the medium, either by actually emitting these fields into the real medium or by processing them in the computer. The aim of this paper is to discuss a number of subtle differences between the focusing conditions in the various methods and to point out the causality/acausality aspects of the corresponding focusing wavefields.
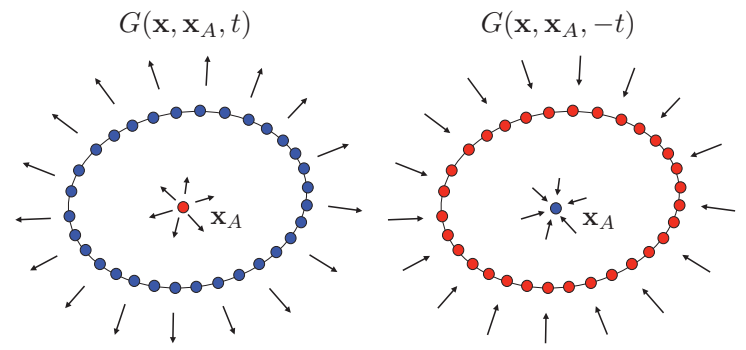

Fig. 1: Principle of time-reversed acoustics.

\section{FOCUSING BY TIME-REVERSAL}

Time-reversed acoustics, as advocated by Fink (1997) and coworkers, employs the invariance of the wave equation for time-reversal (assuming the medium is lossless). The principle is illustrated in Figure 1. A wavefield $G\left(\mathbf{x}, \mathbf{x}_{A}, t\right)$, recorded on a boundary enclosing the source at $\mathbf{x}_{A}$ in an arbitrary inhomogeneous medium, is reversed in time and emitted from the boundary into the medium. At $t=0$ the field focuses at $\mathbf{x}_{A}$. Because there is no sink to absorb the field, the focal point acts as a virtual source. This is illustrated with a $1 \mathrm{D}$ example in Figure 2. A source is present at $z=3600 \mathrm{~m}$ in a horizontally layered medium. The top- and bottom-frame in Figure 2 are the time-reversed Green's functions, emitted into

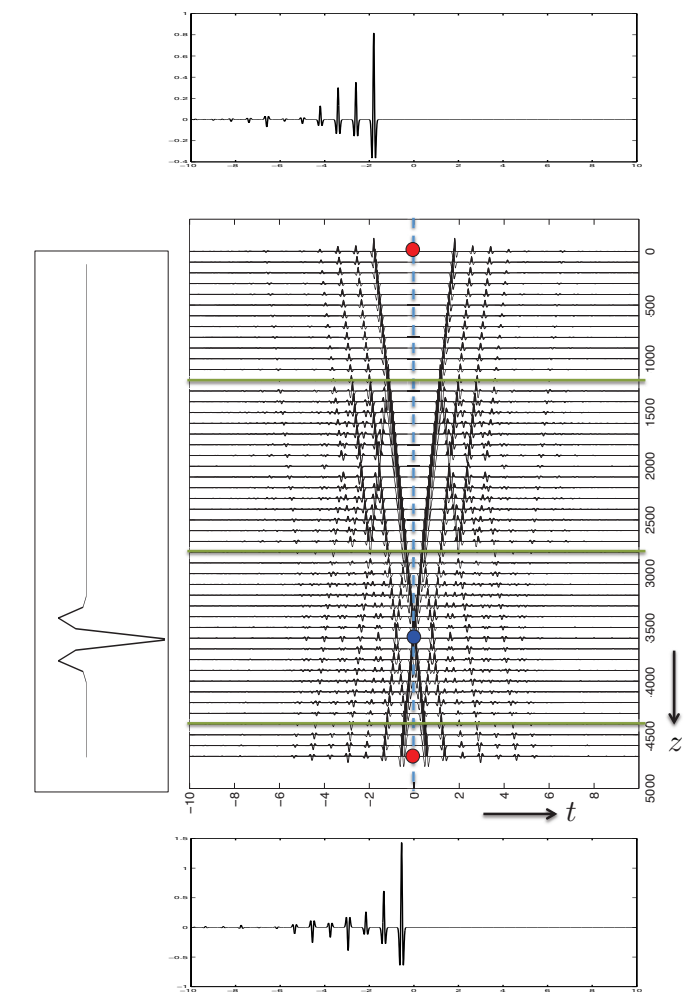

Fig. 2: Time-reversed acoustics in a $1 D$ medium.

the medium from the upper and lower boundary. The middle frame shows the propagation of these wavefields through the layered medium. The field focuses at $t=0$ at the blue dot; the left frame shows the focused field at $t=0$. The response right of the dashed blue line $(t>0)$ can be seen as the response to a virtual source at the blue dot.

We formulate the focusing condition for the 3D situation (Figure 1) as:

- Type-1 focusing: at $t=0$ the focus is proportional to a bandlimited version of $\delta\left(x-x_{A}\right) \delta\left(y-y_{A}\right) \delta\left(z-z_{A}\right)$.

For the 1D situation (Figure 2, left frame) this simplifies to

- Type-1 focusing: at $t=0$ the focus is proportional to a bandlimited version of $\delta\left(z-z_{A}\right)$.

In many practical situations the wavefields are recorded only on one side of the medium, thus violating the closed boundary configuration illustrated in Figure 1. Despite this violation, multiple scattered energy contained in a 3D recorded wavefield contributes significantly to the spatial resolution that can be achieved with time-reversed acoustics (Snieder and Scales, 1998; Fink and Prada, 2001). Nevertheless, single-sided focusing with time-reversed wavefields is far from ideal. This is illustrated with the 1D example in Figure 3. The time-reversed 


\section{Focusing conditions}
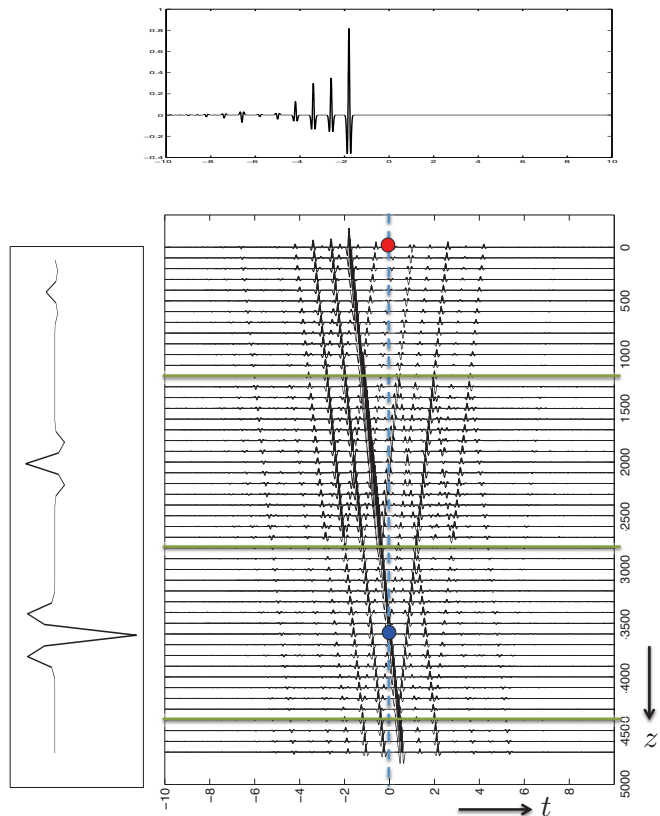

Fig. 3: Time-reversed acoustics with single-sided illumination.

Green's function is only emitted from the upper boundary into the medium. At $t=0$, the wavefield focuses at the blue dot, but also at other depths (left frame). The response right of the dashed blue line $(t>0)$ is no longer the exact response to a virtual source at the blue dot.

Standard seismic interferometry is closely related to timereversed acoustics. Instead of actually emitting a time-reversed field into the medium, recorded wavefields are crosscorrelated, but the results are very similar (Derode et al., 2003; Wapenaar et al., 2005; van Manen et al., 2005; Bakulin and Calvert, 2006). Hence, seismic interferometry suffers from the same limitations in the case of one-sided acquisition (Snieder et al. 2006).

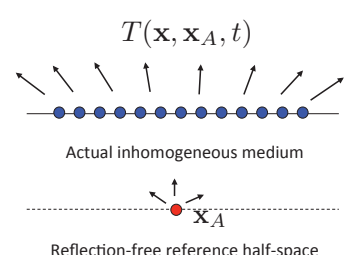

Reflection-free reference half-space

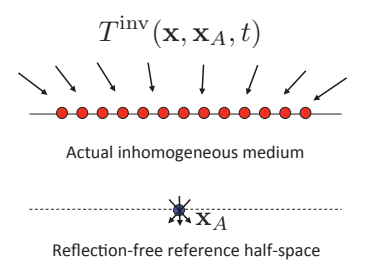

Fig. 4: Focusing by inversion.

\section{FOCUSING BY INVERSION}

Consider the single-sided acquisition configuration shown in Figure 4 . The transmission response $T\left(\mathbf{x}, \mathbf{x}_{A}, t\right)$ is defined as the response to the source at $\mathbf{x}_{A}$, observed at the acquisition surface, in a configuration in which the medium below $\mathbf{x}_{A}$ is reflection-free. By emitting the inverse of this transmission response into the medium, focusing occurs at depth level $z_{A}$.
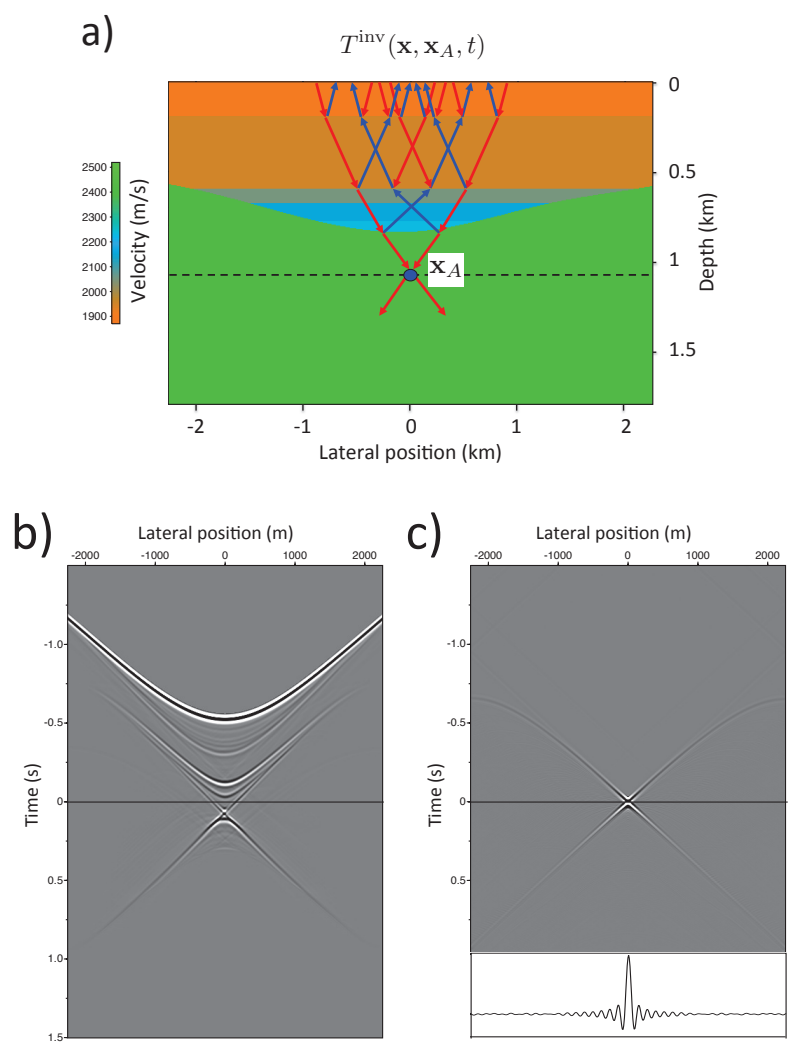

Fig. 5: Focusing with the inverse transmission response. (a) Ray diagram of the inverse transmission response. (b) The inverse transmission response which is emitted into the reference configuration. (c) Its response at the focal depth. Inset: cross-section of the focus at the central frequency.

We formulate this as:

- Type-2 focusing: at $z=z_{A}$ the focus is proportional to a bandlimited version of $\delta\left(x-x_{A}\right) \delta\left(y-y_{A}\right) \delta(t)$.

This is illustrated in Figure 5. For the 1D situation this condition simplifies to

- Type-2 focusing: at $z=z_{A}$ the focus is proportional to a bandlimited version of $\delta(t)$.

This is illustrated in Figure 6. The top-frame shows the inverse transmission response, emitted into the medium from the upper boundary. Note the difference with the time-reversed Green's function in the top-frame of Figure 2. In both cases, the strong event at $t=-1.8 \mathrm{~s}$ is the time-reversed direct arrival. However, whereas in Figure 2 the coda is purely acausal (i.e., preceding the time-reversed direct arrival), the coda in Figure 6 is purely causal (i.e., it follows the time-reversed direct arrival; in this specific example the coda consists of a single event only). The explanation for the causality of this coda is simple: the transmission response of a layered medium is causal and minimum-phase (Anstey and O'Doherty, 1971), and the inverse of a minimum-phase signal is causal and minimum-phase as well (Robinson, 1954) (this causality argument holds for the 1D case; for 3D media it does not hold in a strict sense (Vasconcelos et al., 2014)). The middle frame in Figure 6 shows the propagation of the inverse transmission response through the layered medium. The bottom frame shows the response at 


\section{Focusing conditions}
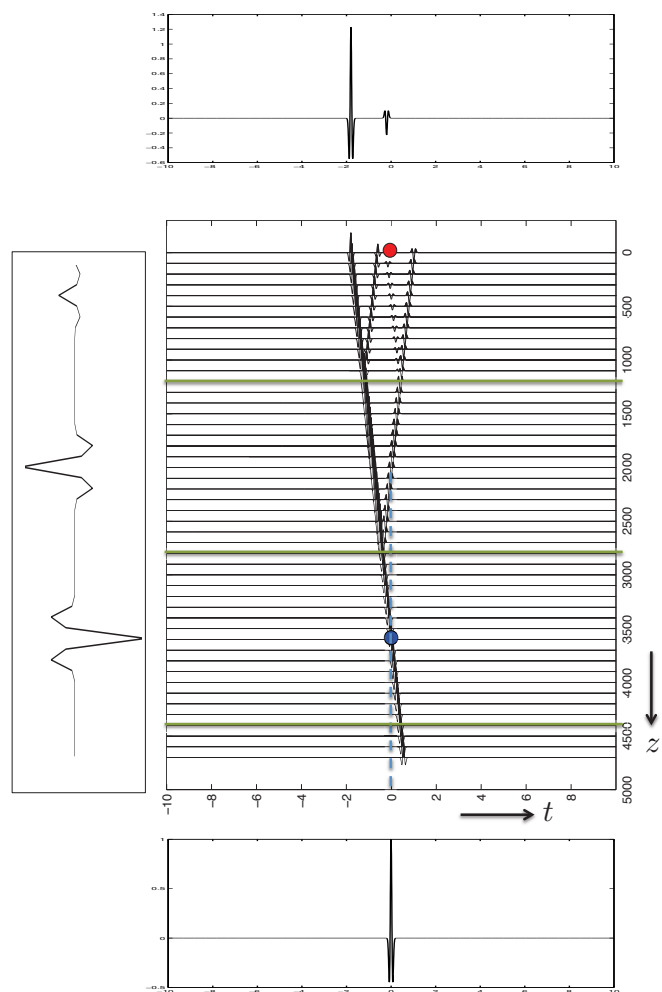

Fig. 6: Focusing by emitting the inverse transmission response into the reference configuration (Figure 4).

the focal depth $z=z_{A}$, which indeed reveals a bandlimited version of $\delta(t)$. The left-frame shows the field at $t=0$, which does not show a unique focus.

The comparison between Figures 2 and 6 reveals the differences between focusing by time-reversal and focusing by inversion:

- Omnidirectional versus single-sided illumination,

- Focusing function with acausal versus causal coda,

- Focusing occurs at $t=0$ (type-1) versus at $z=z_{A}$ (type-2),

- The virtual source at the focal point is omnidirectional, versus radiating downward only.

Focusing by inversion requires knowledge of the (inverse) transmission response. When receivers are present in a horizontal borehole and sources at the surface, then, by virtue of reciprocity, the transmission response $T\left(\mathbf{x}, \mathbf{x}_{A}, t\right)$ is measured. Seismic interferometry by multidimensional deconvolution (MDD) (Wapenaar et al., 2011; van der Neut et al., 2011) inverts the measured transmission response and applies this inverse to the reflection data measured in the borehole. Hence, interferometry by MDD accomplishes focusing by inversion as described above.

A more recent application of focusing by inversion is Marchenko imaging. In this methodology the inverse transmission response is retrieved directly from the reflection response at the surface and an estimate of the direct arrival (Wapenaar et al., 2014; Slob et al., 2014). The retrieved inverse transmission response is actually a focusing operator, called $f_{1}^{+}\left(\mathbf{x}, \mathbf{x}_{A}, t\right)$. Its response is $f_{1}^{-}\left(\mathbf{x}, \mathbf{x}_{A}, t\right)$ (the upgoing waves
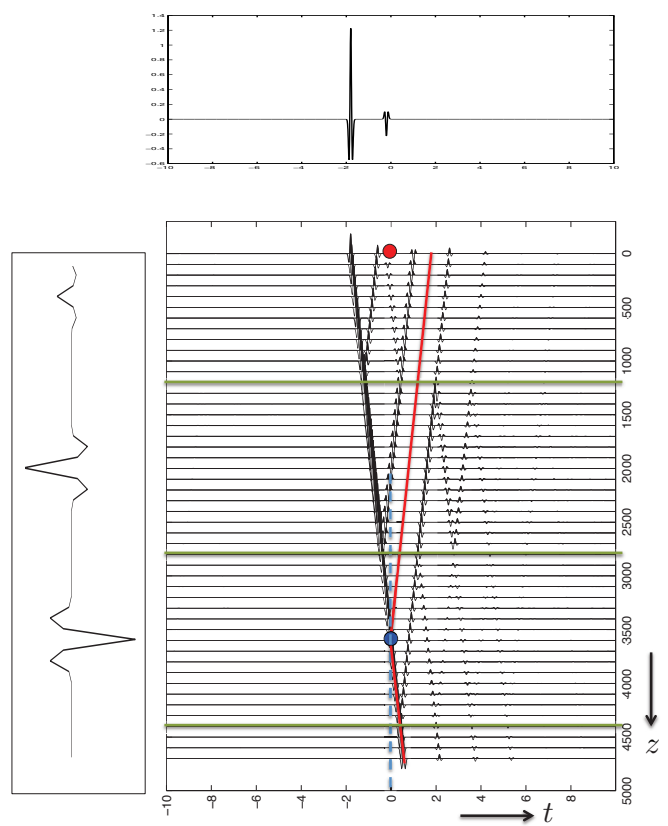

Fig. 7: Focusing function $f_{1}^{+}$(the inverse transmission response) emitted from above into the actual medium. The focal point (blue dot) acts as a virtual source for downgoing waves. The red line separates the focusing function from the decomposed Green's function.

in Figure 6). When the focusing operator $f_{1}^{+}\left(\mathbf{x}, \mathbf{x}_{A}, t\right)$ is emitted into the actual medium (instead of the reference configuration of Figure 4), the field emitted by the downward radiating virtual source at the focal point continues its propagation through the actual medium, see Figure 7 . The red lines in this figure represent the traveltime of the direct arrivals. The response at and beyond the red line is the decomposed Green's function, related to the downward radiating virtual source (the response before the red line is again $\left.f_{1}^{-}\left(\mathbf{x}, \mathbf{x}_{A}, t\right)\right)$. Instead of really emitting the focusing function into the medium, it is convolved with the reflection response at the acquisition surface $\partial \mathbb{D}_{0}$, according to

$$
\begin{aligned}
& G^{p,+}\left(\mathbf{x}, \mathbf{x}_{A}, t\right)+f_{1}^{-}\left(\mathbf{x}, \mathbf{x}_{A}, t\right) \\
& =\int_{\partial \mathbb{D}_{0}} \mathrm{~d} \mathbf{x}^{\prime} \int_{-\infty}^{t} R\left(\mathbf{x}, \mathbf{x}^{\prime}, t-t^{\prime}\right) f_{1}^{+}\left(\mathbf{x}^{\prime}, \mathbf{x}_{A}, t^{\prime}\right) \mathrm{d} t^{\prime},
\end{aligned}
$$

with $\mathbf{x}$ at $\partial \mathbb{D}_{0}$. The Green's function $G^{p,+}\left(\mathbf{x}, \mathbf{x}_{A}, t\right)$ at the left-hand side represents the acoustic pressure $(p)$ at $\mathbf{x}$ at the surface, due to the downward $(+)$ radiating source at $\mathbf{x}_{A}$. Using equation 1 , it is obtained without explicit knowledge of the reflectors in the medium.

The Green's function $G^{p,-}\left(\mathbf{x}, \mathbf{x}_{A}, t\right)$ due to an upward (-) radiating source at $\mathbf{x}_{A}$ is obtained by emitting the time-reversal of $f_{1}^{-}\left(\mathbf{x}, \mathbf{x}_{A}, t\right)$ into the medium (Wapenaar et al., 2014; Slob et al., 2014). Again, instead of really emitting the focusing function into the medium, it is convolved with the reflection response, according to

$$
\begin{aligned}
& G^{p,-}\left(\mathbf{x}, \mathbf{x}_{A}, t\right)-f_{1}^{+}\left(\mathbf{x}, \mathbf{x}_{A},-t\right) \\
& =-\int_{\partial \mathbb{D}_{0}} \mathrm{~d} \mathbf{x}^{\prime} \int_{-\infty}^{t} R\left(\mathbf{x}, \mathbf{x}^{\prime}, t-t^{\prime}\right) f_{1}^{-}\left(\mathbf{x}^{\prime}, \mathbf{x}_{A},-t^{\prime}\right) \mathrm{d} t^{\prime}
\end{aligned}
$$




\section{Focusing conditions}
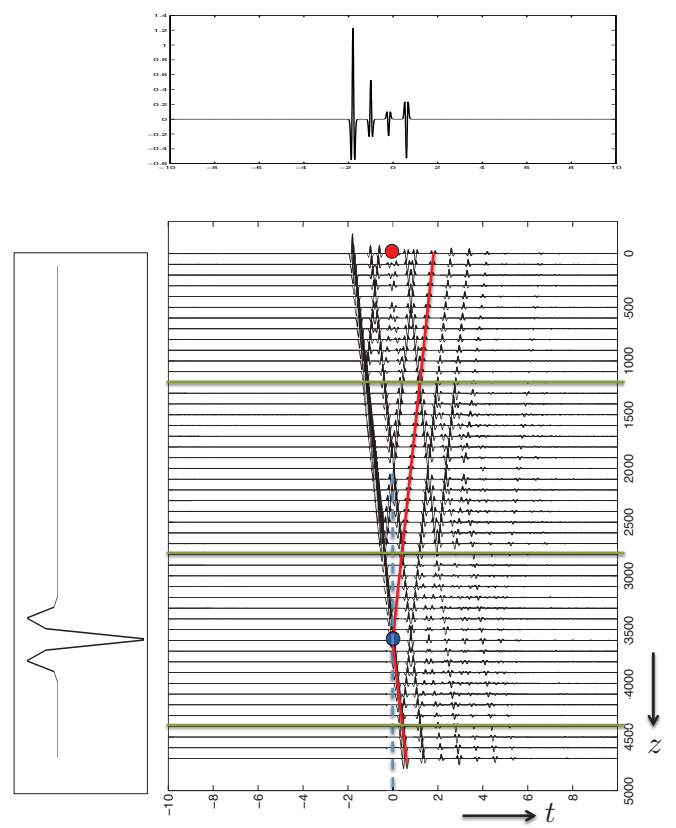

Fig. 8: Sum of equations 1 and 2. The field beyond the red line represents the total Green's function, see also Figure 2. $t=0$ the focus is proportional to a bandlimited version of $\delta\left(x-x_{A}\right) \delta\left(y-y_{A}\right) \delta\left(z-z_{A}\right)$. In practice a medium can often be accessed from one side only, which means that at $t=0$, apart from the desired focus, other foci will occur. Standard seismic interferometry (by crosscorrelation) is based on similar assumptions as time-reversed acoustics and hence suffers from similar limitations in case of single-sided acquisition.

We showed that, in case of single-sided acquisition, the focusing operator is ideally formulated as the inverse of the transmission response. Opposed to the acausal coda in the timereversed focusing operator, the coda of the inverse transmission response is causal (this is true for the 1D case but does not hold strictly for 3D situations). The condition for focusing with the inverse transmission response, which we call type2 focusing, is that at $z=z_{A}$ the focus is proportional to a bandlimited version of $\delta\left(x-x_{A}\right) \delta\left(y-y_{A}\right) \delta(t)$. Single-sided type- 2 focusing properly accounts for multiple scattering. It has applications in seismic interferometry by multidimensional deconvolution, in which the transmission response is measured, and in Marchenko imaging, in which the inverse transmission response is retrieved from the reflection data and an estimate of the direct arrival. In both applications this leads to decomposed Green's functions, which can be used for imaging, properly accounting for multiple reflections.

When the schemes for the decomposed Green's functions are combined we obtain again type- 1 focusing, but a with focusing operator with a causal coda.

with $\mathbf{x}$ at $\partial \mathbb{D}_{0}$.

The decomposed Green's functions can be used for imaging, properly accounting for internal multiple reflections (Wapenaar et al., 2014; Broggini et al., 2014; Behura et al., 2014 Vasconcelos et al., 2014).

Finally, we sum the results of equations 1 and 2, see Figure 8. The upper frame shows the total focusing function $f_{2}\left(\mathbf{x}_{A}, \mathbf{x}, t\right)=f_{1}^{+}\left(\mathbf{x}, \mathbf{x}_{A}, t\right)-f_{1}^{-}\left(\mathbf{x}, \mathbf{x}_{A},-t\right)$, which is emitted into the medium. The middle frame shows how the total focusing function $f_{2}\left(\mathbf{x}_{A}, \mathbf{x}, t\right)$ propagates through the medium and focuses at $t=0$ at the blue dot. The response beyond the red line is the total Green's function, identical to the one obtained with the time-reversal approach in Figure 2. Note that this time we obtained type- 1 focusing using a focusing operator with a causal coda.

The total focusing function $f_{2}\left(\mathbf{x}_{A}, \mathbf{x}, t\right)$ can also be obtained directly from the Marchenko equation (i.e., without decomposition and resolving $f_{1}^{+}$and $f_{1}^{-}$separately). Rose $(2001,2002)$ made the connection between this solution of the Marchenko equation and autofocusing, and Broggini and Snieder (2012) and Broggini et al. (2012) showed that this leads to retrieving the total Green's function.

\section{CONCLUSIONS}

We have shown that, next to the similarities between timereversed acoustics, seismic interferometry and Marchenko imaging, the focusing functions in these methods exhibit a number of subtle differences. Time-reversed acoustics is based on the assumption that the medium of investigation can be accessed from all sides. By emitting the time-reversed response observed at the boundary back into the medium, focusing occurs at the original source position. The condition for this type of focusing, which we call type- 1 focusing, is that at 


\section{Focusing conditions}

\section{References}

Anstey, N. A. and R. F. O'Doherty, 1971, Reflections on amplitudes: Geophysical Prospecting, 19, 430-458.

Bakulin, A. and R. Calvert, 2006, The virtual source method: Theory and case study: Geophysics, 71, SI139-SI150.

Behura, J., R. Snieder, and K. Wapenaar, 2014, Autofocus imaging: Image reconstruction based on inverse scattering theory: Geophysics, 79, (in press).

Broggini, F. and R. Snieder, 2012, Connection of scattering principles: a visual and mathematical tour: European Journal of Physics, 33, 593-613.

Broggini, F., R. Snieder, and K. Wapenaar, 2012, Focusing the wavefield inside an unknown 1D medium: Beyond seismic interferometry: Geophysics, 77, A25-A28.

— , 2014, Data-driven wave field focusing and imaging with multidimensional deconvolution: Numerical examples for reflection data with internal multiples: Geophysics, 79, (in press).

Derode, A., E. Larose, M. Tanter, J. de Rosny, A. Tourin, M. Campillo, and M. Fink, 2003, Recovering the Green's function from field-field correlations in an open scattering medium (L): Journal of the Acoustical Society of America, 113, 2973-2976.

Fink, M., 1997, Time reversed acoustics: Physics Today, 50, 34-40.

Fink, M. and C. Prada, 2001, Acoustic time-reversal mirrors: Inverse Problems, 17, R1-R38.

Robinson, E. A., 1954, Predictive decomposition of seismic traces with applications to seismic exploration: PhD thesis, Massachusetts Institute of Technology.

Rose, J. H., 2001, "Single-sided" focusing of the time-dependent Schrödinger equation: Physical Review A, 65, 012707.

— 2002, 'Single-sided' autofocusing of sound in layered materials: Inverse Problems, 18, 1923-1934.

Slob, E., K. Wapenaar, F. Broggini, and R. Snieder, 2014, Seismic reflector imaging using internal multiples with Marchenko-type equations: Geophysics, 79, S63-S76.

Snieder, R., K. Wapenaar, and K. Larner, 2006, Spurious multiples in seismic interferometry of primaries: Geophysics, 71, SI111-SI124.

Snieder, R. K. and J. A. Scales, 1998, Time-reversed imaging as a diagnostic of wave and particle chaos: Physical Review E, 58, 5668-5675.

van der Neut, J., J. Thorbecke, K. Mehta, E. Slob, and K. Wapenaar, 2011, Controlled-source interferometric redatuming by crosscorrelation and multidimensional deconvolution in elastic media: Geophysics, 76, SA63-SA76.

van Manen, D.-J., J. O. A. Robertsson, and A. Curtis, 2005, Modeling of wave propagation in inhomogeneous media: Physical Review Letters, 94, 164301.

Vasconcelos, I., D.-J. van Manen, M. Ravasi, K. Wapenaar, and J. van der Neut, 2014, Marchenko redatuming: advantages and limitations in complex media: 84th Annual International Meeting, Expanded Abstracts, (this conference.

Wapenaar, K., F. Broggini, and R. Snieder, 2012, Creating a virtual source inside a medium from reflection data: heuristic derivation and stationary-phase analysis: Geophysical Journal International, 190, 1020-1024.

Wapenaar, K., J. Fokkema, and R. Snieder, 2005, Retrieving the Green's function in an open system by crosscorrelation: a comparison of approaches (L): Journal of the Acoustical Society of America, 118, 2783-2786.

Wapenaar, K., J. Thorbecke, J. van der Neut, F. Broggini, E. Slob, and R. Snieder, 2014, Marchenko imaging: Geophysics, 79, (in press).

Wapenaar, K., J. van der Neut, E. Ruigrok, D. Draganov, J. Hunziker, E. Slob, J. Thorbecke, and R. Snieder, 2011, Seismic interferometry by crosscorrelation and by multidimensional deconvolution: a systematic comparison: Geophysical Journal International, 185, 1335-1364. 